\title{
Fungal peritonitis in patients undergoing continuous ambulatory peritoneal dialysis in Qatar
}

\author{
Fahmi Y. Khan ${ }^{1}$, Mohammed Elsayed ${ }^{2}$, Deshmukh Anand ${ }^{3}$, Mohammed Abu Khattab ${ }^{4}$, \\ Doiphode Sanjay ${ }^{3}$
}

Department of Medicine, General Medicine ${ }^{1}$, Nephrology Division ${ }^{2}$, Infectious Diseases Division $^{4}$ and Department of Microbiology ${ }^{3}$, Hamad General Hospital, Doha, Qatar

\begin{abstract}
Introduction: This study was conducted at Hamad General Hospital to determine the incidence of fungal peritonitis and to describe its clinical and microbiological findings in patients undergoing continuous ambulatory peritoneal dialysis in Qatar.

Methodology: The medical records of these patients between 1 January 2005 and 31 December 2008 were retrospectively reviewed and the collected data were analysed.

Results: During the study period, 141 episodes of peritonitis were observed among 294 patients. In 14 of these episodes (9.9\%), fungal peritonitis was reported in 14 patients with a rate of 0.05 episodes per patient year, while the bacterial peritonitis rate was 0.63 per patient year. Thirteen $(93 \%)$ patients had one or more previous episodes of bacterial peritonitis that was treated with multiple broad-spectrum antibiotics, $11(85 \%)$ had received broad-spectrum antibiotics within the preceding month, $12(92 \%)$ within three months, and $8(62 \%)$ within six months. Candida species were the only fungal species isolated from the dialysate with predominance of non-albicans Candida species (especially Candida parapsilosis). Therapeutic approach was immediate catheter removal, followed by systemic antifungal therapy and temporary haemodialysis. Nine patients $(64.3 \%)$ were continued on haemodialysis, whereas five patients $(35.7 \%)$ died.

Conclusions: Prior antibiotic use was an important risk factor predisposing patients to the development of fungal peritonitis. Early detection of fungal peritonitis would lead to early institution of appropriate therapy and prevention of complications.
\end{abstract}

Key words: amphotericin B; CAPD; Candida parapsilosis; fungal peritonitis; fluconazole

J Infect Dev Ctries 2011; 5(9):646-651.

(Received 24 August 2010 - Accepted 29 November 2010)

Copyright (C) 2011 Khan et al. This is an open-access article distributed under the Creative Commons Attribution License, which permits unrestricted use, distribution, and reproduction in any medium, provided the original work is properly cited.

\section{Introduction}

Since its inception in 1976, continuous ambulatory peritoneal dialysis (CAPD) has been an effective treatment for end-stage renal disease (ESRD). In the United States, the treatment of $13 \%$ of patients requiring long-term renal substitution is managed with CAPD, [1] whereas in Qatar 22\% of the ESRD population is treated with CAPD [2].

In spite of technical improvements in CAPD treatment, peritonitis remains one of the most frequent complications of this dialysis modality. The causative organisms are usually bacteria [3]. Fungi are uncommonly implicated, being responsible for only $2-16 \%$ of all peritonitis episodes associated with CAPD [4-12]. Fungal peritonitis, if not detected early and treated appropriately, is associated with increased morbidity (development of peritoneal adhesions and inability to resume CAPD) and mortality. The mortality rate varies from $5 \%$ to $53 \%$, while failure to resume CAPD occurs in up to $40 \%$ of the patients [612].

There is a lack of information about the clinical pattern of fungal peritonitis in patients undergoing CAPD in most Arab countries. This prompted us to conduct this retrospective study to determine the incidence and to describe the clinical findings, fungal species, treatment, and outcome of fungal peritonitis in patients with ESRD undergoing CAPD in Qatar.

\section{Methodology}

In Qatar, haemodialysis is provided by Hamad General Hospital's main dialysis unit and its satellite units, namely the Al Wakra and Alkor dialysis units. The three units harbour a total of 37, 11, and 6 dialysis stations respectively; operating a total of three shifts per day. On the other hand, peritoneal dialysis is provided by the peritoneal dialysis unit at Al-Rumaillah Hospital. All patients with suspected 
peritonitis are admitted in the medical ward at Hamad General Hospital for management.

From 1 January 2005 to 31 December 2008, a total of 294 patients with ESRD had been treated with CAPD at the peritoneal dialysis unit. Medical records of all patients with fungal peritonitis during this period were retrospectively reviewed and studied.

Diagnostic criteria for fungal peritonitis included 1) cloudy effluent containing a white cell count $\geq$ $100 / \mu \mathrm{l}$ with a differential count of $50 \%$ polymorphonuclear cells, and 2) isolation of fungus from the peritoneal dialysis effluent or catheter tip on one or more occasions.

The processing of the peritoneal dialysis effluent sample included a leukocyte count, centrifugation of cloudy effluent, and Gram and Giemsa staining of the pellet (i.e., the sediment of peritoneal dialysis effluent), which was resuspended in $5 \mathrm{ml}$ of sterile saline. Next $2 \mathrm{ml}$ of suspension was inoculated in BACTEC Peds Plus/F media (BD BACTEC FX Blood Culture Instrument) and the remaining suspension was inoculated on sheep blood agar, chocolate agar and MacConkey agar for aerobic culture and on Schindler's agar for anaerobic culture, at $37^{\circ} \mathrm{C}$ incubation. Fungal cultures were performed by inoculating the suspension on Sabouraud's dextrose agar. All the bacterial isolates were identified, and antimicrobial susceptibility testing was performed using the Phoenix Automated Microbiology System (BD Diagnostic Systems, Sparks, MD, USA). Candida was identified using the YST ID Card (Vitek 2 Compact, bioMérieux, Marcy l'Etoile, France) and antifungal susceptibility testing done using the E-test for amphotericin B, 5fluorocytosine, fluconazole, Iiraconazole and ketoconazole.

The current guidelines for the treatment of fungal peritonitis in our centre include immediate catheter removal and administration of a combination of intravenous amphotericin B (20 mg once daily) and oral fluconazole (100 mg once daily) while awaiting results of sensitivity. The treatment continues for two weeks if the fungal isolates are sensitive to these drugs.

Demographic features, clinical manifestations, dialysate analysis and outcome were reviewed, and the following specific data were recorded: age, gender, cause of ESRD, type of peritoneal dialysis, presence of comorbid diseases, number of months on the peritoneal dialysis program, prior history of bacterial peritonitis, antibiotic use within three months before fungal peritonitis, presence of fungaemia, signs and symptoms of peritonitis, peritoneal fluid white blood cells (WBC) count (and percentage of neutrophils), Gram stain and culture, fungus species causing peritonitis, type of antifungal agents administered and outcome.

The gathered information was transferred to a computer utilizing EpiInfo 2000 software. Data were analysed using simple descriptive statistics. The study was approved by the research committee of the Hamad Medical Corporation.

\section{Results}

During the four-year study period, 141 episodes of peritonitis were observed among 294 patients undergoing CAPD in our centre. In 14 of these episodes $(9.9 \%)$, fungal peritonitis was reported in 14 patients. Seven episodes developed in 2005, two in 2006, two in 2007 and three in 2008. Of the 14 patients, $11(78.6 \%)$ were males and three $(21.4 \%)$ were females. The mean age was $50.4 \pm 21.7$ years (range of 1-70 years). Twelve of the patients (85.7\%) were non-Qataris, whereas two (14.3\%) were Qataris.

The peritonitis rate in patients who developed fungal infection was 0.05 episodes per patient year, while it was 0.63 per patient year in those patients with bacterial peritonitis.

Clinical picture, dialysate analysis, and fungal species

The mean duration of CAPD was $19.5 \pm 9$ months (range of 6-36 months). The primary renal diseases leading to ESRD were diabetic nephropathy in eight $(57.1 \%)$ patients and chronic glomerulonephritis in three $(21.4 \%)$ patients and hypertensive nephropathy in one $(7.2 \%)$ patient. In two patients (14.3\%), the exact etiology of the primary renal disease was not clear. Table 1 describes the demographic data, etiology of ESRD, and clinical characteristics of 14 cases of fungal peritonitis in CAPD patients. Thirteen (93\%) patients had one or more previous episode of bacterial peritonitis that was treated with multiple broad-spectrum antibiotics; $11(85 \%)$ of the fungal peritonitis patients had received broad-spectrum antibiotics within the preceding month, 12 (92\%) within three months, and eight (62\%) within six months.

The common clinical presentation was abdominal pain and fever. Table 2 describes the clinical presentation of patients with fungal peritonitis.

The Y-set disconnect system was used in all 14 patients. The mean dialysate white cell count was 
Table 1. Demographic data, etiology of ESRD and clinical characteristics of 14 cases with fungal peritonitis in CAPD patients

\begin{tabular}{|l|c|}
\hline Variable & Number of patients (\%) \\
\hline Age [Mean \pm SD years] & $50.4 \pm 21.7$ years \\
Sex (M:F) & $3.6: 1$ \\
Mean duration of CAPD (months) & $19.5 \pm 9$ months \\
Etiology of ESRD & \\
Diabetic nephropathy & $8(57.1 \%)$ \\
Chronic glomerulonephritis & $3(21.4 \%)$ \\
Hypertensive nephropathy & $1(7.2 \%)$ \\
Unknown & $2(14.3 \%)$ \\
Dialysis exchange system used & $14(100 \%)$ \\
Y-set & $1546 \pm 1956 / \mu 1$ \\
Mean dialysate white cell count/ $\boldsymbol{\mu l}$ & $80 \% \pm 14.8 \%$ \\
Neutrophils (\%) [Mean \pm SD] & $13(93 \%)$ \\
Recent antibiotic therapy before FP & $14(100 \%)$ \\
Catheter removal & $5(35.7 \%)$ \\
Mortality & \\
\hline FP: fungal peritonitis, CAPD: continuous ambulatory peritoneal dialysis ESRD: end stage renal \\
\end{tabular}

Table 2. Common presentation of fungal peritonitis in 14 CAPD patients

\begin{tabular}{|l|l|}
\hline Clinical presentation & Number of patients (\%) \\
\hline Abdominal pain & $11(78.6 \%)$ \\
Fever & $10(71.4 \%)$ \\
Nausea \& vomiting & $7(50 \%)$ \\
Inflow-outflow problem & $7(50 \%)$ \\
Ultra-filtration failure & $4(28.6 \%)$ \\
\hline
\end{tabular}

Table 3. Species of fungi isolated during 14 episodes of fungal peritonitis in CAPD patients

\begin{tabular}{|l|l|}
\hline Causative agent (S) & Number of episodes (\%) \\
\hline $\begin{array}{l}\text { Candida albicans } \\
\text { Non-albicans Candida species }\end{array}$ & $2(14.3 \%)$ \\
$\bullet \quad$ Candida parapsilosis & $8(57.1 \%)$ \\
- Candida tropicalis & $3(21.4 \%)$ \\
- Candida krusei & $1(7.2 \%)$ \\
\hline
\end{tabular}


$1546 \pm 1956 / \mu 1$, with $80 \% \pm 14.8 \%$ neutrophils. Only Candida species were isolated from the dialysate of our patients. All of them were sensitive to amphotericin B, fluconazole, 5-fluorocytosine, itraconazole, and ketoconazole. Non-albicans Candida species as a group (85.7\%) was more common than Candida albicans (14.3\%). Nonalbicans Candida species included Candida parapsilosis 8 (57.1\%), Candida tropicalis 3 (21.4\%) and Candida krusei 1 (7.2\%) (Table3). Concomitant candidaemia (Candida albicans and Candida parapsilosis) was found in two (14.3\%) patients.

\section{Treatment and outcome}

All patients were treated with immediate CAPD catheter removal within 24 hours of the diagnosis of fungal peritonitis, followed by systemic antifungal therapy. All patients received empirical intravenous amphotericin B (20 mg once daily) and oral fluconazole (200 $\mathrm{mg}$ once daily) while awaiting results of sensitivity, and they were temporarily initiated on haemodialysis. All fungal isolates were susceptible to amphotericin B and fluconazole, which were continued for two weeks. Nine patients $(64.3 \%)$ were continued on haemodialysis, whereas five patients $(35.7 \%)$ died during the episode of fungal peritonitis.

\section{Discussion}

In this study, male patients predominated regardless of their nationality, which seems to be artificial and can be explained by the fact that Qatar and other Gulf countries have a large working community composed mainly of males.

As noted, fungi accounted for $9.9 \%$ of all peritonitis episodes in our study, which falls within the reported range of $2-16 \%$ described in the literature [4-12]. Fungi enter the peritoneal cavity through touch contamination at the time of PD exchange or invasion of skin by the offending organisms from the exit site through the tunnel to the peritoneum [13]. Therefore, occurrence of fungal peritonitis in our patients could be explained by the inability of our patients to adopt good aseptic practices, which emphasizes the need to improve the training of our patients in aseptic CAPD technique, especially in hand washing technique and in making the connection. There is controversy regarding the predisposing factors for fungal peritonitis in patients undergoing CAPD. Rupture of the cutaneous barrier, as a result of the presence of the catheter, and reduced cellular immunity during chronic end-stage renal disease were suggested to favor the occurrence of fungal peritonitis in these patients. In addition, many reports have shown that prior antibiotic use is an important risk factor predisposing to the development of fungal peritonitis. The reported incidence of prior antibiotic exposure in CAPD patients with fungal peritonitis ranges from $55-94 \%$ [11-18]. Goldie et al. [18] reported that $65 \%$ of fungal peritonitis patients had received broadspectrum antibiotics within the preceding month, $74 \%$ within three months, and $87 \%$ within six months. In our study, 13 (93\%) patients had one or more previous episodes of bacterial peritonitis that were treated with multiple broad-spectrum antibiotics; 11 (85\%) of the fungal peritonitis patients had received broad-spectrum antibiotics within the preceding month, $12(92 \%)$ within three months, and eight $(62 \%)$ within six months. The previous use of antibiotics for bacterial peritonitis may kill the normal bowel flora and allow fungal proliferation and overgrowth, which migrate across the intestinal wall and invade the inflamed peritoneum. Other important contributors to the increased risk of fungal peritonitis in this study include socioeconomic factors, such as housing conditions, nutritional status and remoteness of living, as environmental exposures have been found to predispose to Candida peritoneal infections [19-21]. It is noteworthy that $83 \%$ of our patients were non-Qataris (mainly from the Indian subcontinent), and were of low socioeconomic conditions.

On the other hand, in many reports as well as in ours, age, sex and type of dialysis exchange system did not correlate to the development of fungal peritonitis. Moreover, the role of diabetes has been variously evaluated in different series, but in most studies it does not seem to predispose patients to fungal peritonitis [4].

Prophylactic antifungal treatment during antibiotic therapy in patients receiving CAPD has been examined by many authors, but the results have been mixed [6]. Despite this, the International Society of Peritoneal Dialysis (ISPD) guidelines recommend that fungal prophylaxis during antibiotic therapy may prevent some cases of Candida peritonitis in programs that have high rates of fungal peritonitis [10]. Although 93\% of our patients had one or more previous episode of bacterial peritonitis that was treated with multiple broad-spectrum antibiotics, none of them had received prophylactic antifungal treatment during antibiotic therapy. This fact might explain partially the reason for the high 
morbidities and mortalities in our study. Therefore, we strongly recommend using fungal prophylaxis during antibiotic therapy in an attempt to reduce morbidities and mortalities in our centre.

The mean duration of CAPD treatment before the diagnosis of fungal peritonitis in our patients $(19.5 \pm$ 9 months) is also similar to previously reported figures [12,14].

Candida species were the most common causative agents for the fungal peritonitis, accounting for $60-100 \%$ of episodes as the sole pathogen [6-24]. In our study, all cases (100\%) of fungal peritonitis were caused by Candida species, which falls within the reported range. In some reports, Candida albicans was reported to be more common than non-albicans Candida species [12,23], whereas other reports disclosed predominance of non-albicans Candida species $[3,4,6,14,16,18,24]$. In our study nonalbicans Candida species (especially Candida parapsilosis) were the most common pathogen. The reason that Candida parapsilosis was so common in our investigation is unclear and needs further study.

Current treatment recommendations from the ISPD include immediate catheter removal after fungi identification by microscopy or culture, and the administration, as initial therapy, of a combination of amphotericin B and 5-flucytosine until culture and susceptibility results become available [10]. In this study, the CAPD catheter was removed from all patients at the time of diagnosis and all of the patients received empirical antifungal therapy (intravenous amphotericin B and oral fluconazole) and were temporarily initiated on haemodialysis.

As reported in the literature, failure to resume CAPD occurs in up to $40 \%$ of the patients. Peritoneal adhesion, sclerosis, and irreversible damage to the peritoneal membrane causing a reduction in solute transport or ultrafiltration remain the major reasons for CAPD dropout. In our study, all patients who survived were continued on haemodialysis, due to failure to resume CAPD. The reason for this high failure rate is not clear and remains to be clarified. It could have resulted from delay in reaching the diagnosis, which emphasizes the need to improve the techniques used in the diagnosis of fungal peritonitis as recommended by the ISPD [25].

Globally, the mortality rate varies from $5-53 \%$. In this study, five patients $(35.7 \%)$ died during the episode of fungal peritonitis, which falls within the global range.
The limitation of this study, as with many studies, is the small number of cases. Moreover, the study was retrospective rather than prospective.

In conclusion, fungal peritonitis is a recognised cause of morbidity and mortality in patients undergoing CAPD in our centre. Prior antibiotic use was an important risk factor predisposing patients to the development of fungal peritonitis. Early detection of fungal peritonitis would lead to early institution of appropriate therapy and prevention of complications (peritoneal adhesions and inability to resume CAPD). We suggest that further prospective studies are needed to confirm these findings and to explain the predominance of Candida parapsilosis and high rate of CAPD dropout.

\section{References}

1. Diaz-Buxo JA (1991) CAPD in North America. Contrib Nephrol 89: 6-10.

2. Fituri OM, Shigidi MM, Ramachandiran G, Rashed AH (2009) Demographic data and hemodialysis population dynamics in Qatar: A five year survey. Saudi J Kidney Dis Transpl 20: 493-500.

3. Ram R, Swarnalatha G, Neela P, Murty KV (2008) Fungal peritonitis in patients on continuous ambulatory peritoneal dialysis: a single-centre experience in India. Nephron Clin Pract 110: 207-212.

4. Bibashi E, Memmos D, Kokolina E, Tsakiris D, Sofianou D, Papadimitriou M (2003) Fungal Peritonitis Complicating Peritoneal Dialysis during an 11-Year Period: Report of 46 Cases. Clin Infect Dis 36 :927-931.

5. Kerr CM, Perfect JR, Craven PC, Jorgensen JH, Drutz DJ, Shelburne JD, Gallis HA, Gutman RA (1983) Fungal peritonitis in patients on continuous ambulatory peritoneal dialysis. Ann Intern Med 99: 334-336.

6. Prasad KN, Prasad N, Gupta A, Sharma RK, Verma AK, Ayyagari A (2004) Fungal peritonitis in patients on continuous ambulatory peritoneal dialysis: a single centre Indian experience. J Infect 48: 96-101.

7. Chan TM, Chan CY, Cheng SW, Lo WK, Lo CY, Cheng IKP (1994) Treatment of fungal peritonitis complicating continuous ambulatory peritoneal dialysis with oral fluconazole: a series of 21 patients. Nephrol Dial Transplant 9: 539-542.

8. Michel C, Courdavault L, al Khayat R, Viron B, Roux P, Mignon F (1994) Fungal peritonitis in patients on peritoneal dialysis. Am J Nephrol 14: 113-120.

9. Nagappan R, Collins JF, Lee WT (1992) Fungal peritonitis in continuous ambulatory peritoneal dialysis: the Auckland experience. Am J Kidney Dis 20: 492-496.

10. Piraino B, Bailie GR, Bernardini J, Boeschoten E, Gupta A, Holmes C, Kuijper EJ, Li PK, Lye WC, Mujais S, Paterson DL, Fontan MP, Ramos A, Schaefer F, Uttley L (2005) Peritoneal Dialysis related infections recommendations: 2005 Update. Perit Dial Int 25: 107-131.

11. Kazancioglu R, Kirikci G, Albaz M, Dolgun R, Ekiz S (2010) Fungal peritonitis among the peritoneal dialysis patients of four Turkish centres. J Ren Care 36: 186-190.

12. Indhumathi E, Chandrasekaran V, Jagadeswaran D, Varadarajan M, Abraham G, Soundararajan P (2009) The risk 
factors and outcome of fungal peritonitis in continuous ambulatory peritoneal dialysis patients. Indian $\mathbf{J}$ Med Microbiol 27: 59-61.

13. Piraino B (1998) Peritonitis as complication of peritoneal dialysis. J Am Soc Nephrol 9: 1956-1964.

14. Chen CM, Ho MW, Yu WL, Wang JH (2004) Fungal peritonitis in peritoneal dialysis patients: effect of fluconazole treatment and use of the twin-bag disconnect system. J Microbiol Immunol Infect 37: 115-20.

15. Johnson RJ, Ramsey PG, Gallagher N, Ahmad S (1985) Fungal peritonitis in patients on CAPD, Incidence, clinical features and prognosis. Am J Nephrol 5: 169-175.

16. Stanley HK, Chan CK (2003) Risk factors for poor outcome of fungal peritonitis on Chinese patients on CAPD. Perit Dial Int 23: 123-126.

17. Prasad N, Gupta A (2005) Fungal peritonitis in peritoneal dialysis patients. Perit Dial Int 25: 207-222.

18. Goldie SJ, Kiernan-Tridle L, Torres C, Gorban-Brennan N, Dunne D, Kliger AS, Finkelstein FO (1996) Fungal peritonitis in a large chronic peritoneal dialysis population: a report of 55 episodes. Am J Kidney Dis 28: 86-91.

19. Miles R, Hawley CM, McDonald SP (2009) Predictors and outcomes of fungal peritonitis in peritoneal dialysis patients. Kidney Int 76: 622-628.

20. Greaves I, Kane K, Richards NT, Elliott TS, Adu D, Michael J (1992) Pigeons and peritonitis? Nephrol Dial Transplant 7: 967-969.

21. Yuen KY, Seto WH, Ching TY, Cheung WC, Kwok Y, Chu YB (1992) An outbreak of Candida tropicalis peritonitis in patients on intermittent peritoneal dialysis. J Hosp Infect 22: 65-72.
22. Molina P, Puchades MJ, Aparicio M, García Ramón R, Miguel A (2005) Fungal peritonitis episodes in a peritoneal dialysis centre during a 10-year period: a report of 11 cases. Nefrologia 25: 393-398.

23. Predari SC, de Paulis AN, Verón D, Zucchini A, Santoianni JE (2007) Fungal peritonitis in patients on peritoneal dialysis: twenty five years of experience in a teaching hospital in Argentina. Rev Argent Microbiol 39: 213-217.

24. Wang AY, Yu AW, Li PK, Lam PK, Leung CB, Lai KN, Lui SF (2000) Factors predicting outcome of fungal peritonitis in peritoneal dialysis: analysis of a 9-year experience of fungal peritonitis in a single centre. Am J Kidney Dis 36: 1183-92.

25. Boeschoten EW, ter Wee PM, Divino J(2006) Peritoneal dialysis-related infections recommendations 2005-an important tool for quality improvement. Nephrol Dial Transplant 21: ii31-ii33.

\section{Corresponding author}

Fahmi Yousef Khan, (MD)

Consultant, Department of Medicine

Hamad General Hospital

PO Box 3050

Doha-Qatar

Telephone: $0974 \quad 55275989$

Fax: $\quad 0974 \quad 44392273$

Email: fakhanqal@yahoo.co.uk

Conflict of interests: No conflict of interests is declared. 